\title{
Limits of near-coloring of sparse graphs
}

\author{
Paul Dorbec* $\quad$ Tomáš Kaiser ${ }^{\dagger} \quad$ Mickael Montassier*‡ André Raspaud*§
}

December 3, 2012

\begin{abstract}
Let $a, b, d$ be non-negative integers. A graph $G$ is $(d, a, b)^{*}$-colorable if its vertex set can be partitioned into $a+b$ sets $D_{1}, \ldots, D_{a}, O_{1}, \ldots, O_{b}$ such that the graph $G\left[D_{i}\right]$ induced by $D_{i}$ has maximum degree at most $d$ for $1 \leq i \leq a$, while the graph $G\left[O_{j}\right]$ induced by $O_{j}$ is an edgeless graph for $1 \leq j \leq b$. In this paper, we give two real-valued functions $\mathfrak{f}$ and $\mathfrak{g}$ such that any graph with maximum average degree at most $\mathfrak{f}(d, a, b)$ is $(d, a, b)^{*}$-colorable, and there exist non- $(d, a, b)^{*}$-colorable graphs with average degree at most $\mathfrak{g}(d, a, b)$. Both these functions converge (from below) to $2 a+b$ when $d$ tends to infinity. This implies that allowing a color to be $d$-improper (i.e. of type $D_{i}$ ) even for a large degree $d$ increases the maximum average degree that guarantees the existence of a valid coloring only by 1 . Using a color of type $D_{i}$ (even with a very large degree $d$ ) is somehow less powerful than using two colors of type $O_{j}$ (two stable sets).
\end{abstract}

\section{Introduction}

A graph $G$ is $\left(d_{1}, \ldots, d_{k}\right)$-colorable if the vertex set of $G$ can be partitioned into subsets $V_{1}, \ldots, V_{k}$ such that the graph $G\left[V_{i}\right]$ induced by the vertices of $V_{i}$ has maximum degree at most $d_{i}$ for all $1 \leq i \leq k$. This notion generalizes those of proper $k$-coloring (when $d_{1}=\ldots=d_{k}=0$ ) and of $d$-improper $k$-coloring (when $d_{1}=\ldots=d_{k}=d \geq 1$ ).

Planar graphs are known to be $(0,0,0,0)$-colorable (Appel and Haken [1, 2]) and $(2,2,2)$ colorable (Cowen et al. [8]; for a list version, see Eaton and Hull [9] or Škrekovski [13]). This last result was improved for planar graphs with large girth (Škrekovski [14]). We recall that the girth of a graph $G$ is the length of a shortest cycle in $G$ (by convention, the girth of $G$ is $\infty$ when $G$ is a forest). Havet and Sereni [12] gave new bounds for graphs with low maximum average degree. The maximum average degree of a $\operatorname{graph} G$, denoted by $\operatorname{mad}(\mathrm{G})$, is the maximum of the average degrees of all subgraphs of $G$, i.e. $\operatorname{mad}(G)=\max \{2|E(H)| /|V(H)|, H \subseteq G\}$.

Theorem 1 (Havet and Sereni [12]) Every graph $G$ with $\operatorname{mad}(G)<k+\frac{k d}{k+d}$ is $d$-improper $k$ colorable (in fact, $d$-improper $k$-choosable), i.e. $(d, \ldots, d)$-colorable (where the tuple is of size $k)$.

Moreover their upper bound on the maximum average degree is asymptotically sharp:

Theorem 2 (Havet and Sereni [12]) There exists a non-d-improper $k$-colorable graph whose maximum average degree tends to $2 k$ when $d$ goes to infinity.

\footnotetext{
*Univ. Bordeaux, LaBRI UMR5800, F-33400 Talence, France CNRS, LaBRI UMR5800, F-33400 Talence, France. E-mail: dorbec, montassi, raspaud@labri.fr

${ }^{\dagger}$ Department of Mathematics and Institute for Theoretical Computer Science (ITI), University of West Bohemia, Univerzitní 8, CZ-306 14 Plzeň, Czech Republic. E-mail: kaisert@kma.zcu.cz, Partially supported by project P202/12/G061 of the Czech Science Foundation

${ }^{\ddagger}$ Partially supported by ANR Project GRATOS - ANR-09-JCJC-0041-01

§Partially supported by ANR-NSC Project GRATEL - ANR-09-blan-0373-01 and NSC99-2923-M-110-001-MY3
} 
The focus has mostly been on $\left(d_{1}, d_{2}\right)$-colorings. Glebov and Zambalaeva [11] proved that every planar graph $G$ with girth at least 16 is $(1,0)$-colorable. Borodin and Ivanova [3] strengthened this result by proving that every graph $G$ with $\operatorname{mad}(G)<\frac{7}{3}$ is $(1,0)$-colorable, which implies that every planar graph $G$ with girth at least 14 is $(1,0)$-colorable. Recently Borodin and Kostochka [7] showed that every graph $G$ with $\operatorname{mad}(G) \leq \frac{12}{5}$ is $(1,0)$-colorable. In particular, it follows that every planar graph $G$ with girth at least 12 is $(1,0)$-colorable. On the other hand, they constructed graphs $G$ with $\operatorname{mad}(G)$ arbitrarily close (from above) to $\frac{12}{5}$ that are not $(1,0)$-colorable; hence their upper bound on the maximum average degree is best possible. As well, Esperet et al. [10] constructed a non$(1,0)$-colorable planar graph with girth 9 ; hence it remains open only whether planar graphs with girth 10 or 11 are $(1,0)$-colorable.

For $(d, 0)$-colorings with $d \geq 2$, Borodin et al. [4] proved the following:

Theorem 3 (Borodin et al. [4]) Let $d \geq 2$ be an integer. Every graph $G$ with $\operatorname{mad}(G)<\frac{3 d+4}{d+2}$ is $(d, 0)$-colorable. Moreover there exists a non- $(d, 0)$-colorable graph $G$ with $\operatorname{mad}(G)=\frac{3 d+4}{d+2}+\frac{1}{d+3}$.

The proof in [4] extends that in [3] but does not work for $d=1$. In Borodin et al. [5], it is proven that every $\operatorname{graph} G$ with $\operatorname{mad}(G)<\frac{10 d+22}{3 d+9}$ is $(d, 1)$-colorable for $d \geq 2$. Finally, for general $\left(d_{1}, d_{2}\right)$-colorings, Borodin et al. [6] gave some sufficient conditions of $\left(d_{1}, d_{2}\right)$-colorability depending on the density of the graphs.

In this paper, we consider the case where each graph $G\left[V_{i}\right](1 \leq i \leq k)$ is either a subgraph with maximum degree at most $d$, or an edgeless graph. In particular, we prove that having for $G\left[V_{i}\right]$ a subgraph with maximum degree at most $d$ even for a large degree $d$ is no more powerful (in terms of mad) than having two edgeless graphs.

Let $d, a, b$ be non-negative integers, with $d>0$. A graph $G$ is $(d, a, b)^{*}$-colorable if the vertex set of $G$ can be partitioned into subsets $D_{1}, \ldots, D_{a}, O_{1}, \ldots, O_{b}$ such that the graph $G\left[D_{i}\right]$ induced by the vertices of $D_{i}(1 \leq i \leq a)$ has maximum degree at most $d$, while the graph $G\left[O_{j}\right]$ induced by the vertices of $O_{j}(1 \leq j \leq b)$ is an edgeless graph. A $(d, a, b)^{*}$-coloring can be looked at as a $(d, \ldots, d, 0, \ldots, 0)$-coloring with $a$ colors of type $D_{i}$ and $b$ colors of type $O_{j}$. We say that we color a vertex with color $D_{i}$ or $O_{j}$ when it should belong to the sets $D_{i}$ or $O_{j}$, respectively. We prove:

Theorem 4 Let $a, b, d$ be integers with $a+b>0$ and $d>0$. Every graph $G$ with $\operatorname{mad}(G)<$ $\mathfrak{f}(d, a, b)$ is $(d, a, b)^{*}$-colorable, where

$$
\mathfrak{f}(d, a, b)=a+b+\frac{d a(a+1)}{(a+d+1)(a+1)+a b} .
$$

Theorem 5 For any positive integers $d, a, b$, there exists a graph $G_{d, a, b}$ which is not $(d, a, b)^{*}$ colorable but is $(d, 0, a+b+1)^{*}$-colorable, and has maximum average degree $\mathfrak{g}(d, a, b)$, where

$$
\mathfrak{g}(d, a, b)=2 a+b-\frac{2}{(d+1)(b+1)-1}+\frac{2 a+2}{(d+1)^{a+1}(b+1)^{a+1}-1} .
$$

Clearly, both functions $\mathfrak{f}$ and $\mathfrak{g}$ tend to $2 a+b$ when $d$ tends to infinity, showing that asymptotically, our bound of $2 a+b$ is tight.

These results are related on the one hand to the work of Havet and Sereni [12] corresponding to the case $b=0$, where the maximum average degree tends to $2 a$ when $d$ goes to infinity, and on the other hand the work of Borodin et al. [4] corresponding to the case $a=1$ and $b=1$, where the maximum average degree tends to 3 when $d$ goes to infinity. However our results do not imply these two results. For these cases, their results are sharper in the sense that (1) the upper bound on the maximum average degree that guarantees the existence of a $(d, a, b)^{*}$-coloring (for $b=0$ and $a=1, b=1$ ) is higher, and (2) the convergence toward $2 a+b$ (for $b=0$ and $a=1, b=1$ ) given by their constructions is quicker.

The remaining part of this note is dedicated to the proofs of Theorems 4 (Section 2) and 5 (Section 3). All technical details are given in Annex. 


\section{Proof of Theorem 4}

Let $G$ be a counterexample to Theorem 4 of minimum order.

\subsection{Structural properties of a minimum counterexample $G$}

Claim 1 The minimum degree of $G$ is at least $a+b$.

PROOF. By contradiction, suppose $G$ contains a vertex $x$ of degree less than $a+b$. By minimality of $G, G-x$ admits a $(d, a, b)^{*}$-coloring. By the pigeonhole principle, one of the colors $D_{1}, \ldots, D_{a}$, $O_{1}, \ldots, O_{b}$ does not appear among the neighbors of $x$, and we can use it to extend the coloring to the whole graph, reaching a contradiction.

In the following, we call a vertex with degree at most $a+b+d-1$ a small vertex; a vertex with degree at least $a+b+d$ and at most $a+b+2 d-1$ a medium vertex, and a vertex of degree at least $a+b+2 d$ a big vertex. Intuitively, whenever a small vertex is colored $D_{i}$ and has a degree too large in this color class, it can be recolored without affecting the $(d, a, b)^{*}$-coloring. The two following claims use this idea.

Claim 2 Every small vertex is adjacent to at least a non-small vertices.

Proof. By contradiction, suppose $G$ contains a vertex $x$ of degree at most $a+b+d-1$ adjacent to at most $a-1$ non-small vertices. By minimality of $G, G-x$ admits a $(d, a, b)^{*}$-coloring. If not all colors appear in the neighborhood of $x$, then we color $x$ properly (i.e. with a color that does not appear on the neighborhood of $x$ ). Otherwise we color $x$ with a color of type $D_{i}$ that does not appear on the non-small vertices, say $D_{1}$. Observe that $D_{1}$ appears at most $d$ times among the neighbors of $x$ (otherwise the degree of $x$ is at least $a+b+d)$. This coloring is not a $(d, a, b)^{*}$-coloring of $G$ only if there is a small neighbor $z$ of $x$ that is colored with $D_{1}$ and has already $d$ neighbors colored with $D_{1}$. But in that case, $z$ being small has at most $a+b-2$ neighbors not colored $D_{1}$, and we can recolor $z$ properly. Finally we obtain a $(d, a, b)^{*}$-coloring of $G$, a contradiction.

Claim 3 Every medium vertex is adjacent to at least a -1 non-small vertices.

Proof. By contradiction, suppose $G$ contains a vertex $x$ of degree $k$ with $a+b+d \leq k \leq$ $a+b+2 d-1$ which is adjacent to at most $a-2$ non-small vertices. By minimality of $G, G-x$ admits a $(d, a, b)^{*}$-coloring. If all the colors do not appear in the neighborhood of $x$, then we color $x$ properly. Otherwise, we want to color $x$ with a color $D_{i}$ that does not appear on the non-small vertices, and that appears at most $d$ times on the small vertices (if necessary, in a second step, we recolor properly the small vertices that see $d+1$ times the color $D_{i}$ in the same way that we did in Claim 2). Suppose that, among the non-small vertices, $x$ sees $n_{D}$ colors of type $D_{i}$. If $x$ cannot be colored as we wanted, it means that $x$ has $d+1$ neighbors of each of the $a-n_{D}$ colors of type $D_{i}$ left. Since it also sees each color of type $O_{j}$, the degree of $x$ is at least $f\left(n_{D}\right)=\left(a-n_{D}\right)(d+1)+n_{I}+b$. This function is decreasing on $n_{D}$ and thus attains the minimum for the upper possible value of $n_{D}$, namely $a-2$. The degree of $x$ is thus at least $f(a-2)=a+b+2 d$, contradicting the value of $k$.

In the following, by a light small vertex we mean a small vertex adjacent to exactly $a$ non-small vertices. The two following claims are based on the idea that a light small vertex colored $O_{i}$ can be recolored without affecting the $(d, a, b)^{*}$-coloring.

Claim 4 A non-small vertex is adjacent to at least b vertices that are not light small.

PROOF. By contradiction. Suppose $G$ contains a non-small vertex $x$ of degree $k$ adjacent to $k-b+1$ light small vertices $y_{1}, \ldots, y_{k-b+1}$. Let $z_{1}, \ldots, z_{b-1}$ be the other neighbors of $x$. By minimality of $G, G-x$ admits a $(d, a, b)^{*}$-coloring. We want to color $x$ with a color of type $O_{j}$ that does not appear on the $z_{i}$ 's, say $O_{1}$. Yet, a vertex $y$ (among $y_{1}, \ldots, y_{k-b+1}$ ) may be already colored with $O_{1}$. 
In that case we recolor $y$ as follows (and reiterate this process while such a $y$ exists). We first try to properly recolor $y$. If it is possible, then we are done. If it is not possible, then notice that all the $a+b$ colors appear in the neighborhood of $y$. In this last case, we recolor $y$ with a color $D_{i}$ that does not appear on the $a-1$ non-small neighbors of $y$ distinct from $x$, say $D_{1}$. Color $D_{1}$ appears at most $d$ times in the neighborhood of $y$ (otherwise the degree of $y$ would be at least $a+b+d$ ). Now, some small neighbor $t$ of $y$ could be colored with $D_{1}$ and have $d+1$ neighbors colored with $D_{1}$ (including $y$ ), but in that case we can properly recolor $t$ as in the preceding proofs. Finally, we obtain a $(d, a, b)^{*}$-coloring of $G$, a contradiction.

The following claim is a refinement of Claim 4 for medium vertices.

Claim 5 A medium vertex is adjacent to at least $a+b-1$ vertices that are not light small.

Proof. By contradiction. Suppose $G$ contains a vertex $x$ of degree $k$ adjacent to $k-(a-1)-b+1$ light small vertices $y_{1}, \ldots, y_{k-(a-1)-b+1}$. Let $z_{1}, \ldots, z_{a+b-2}$ be the other vertices adjacent to $x$. By minimality of $G, G-x$ admits a $(d, a, b)^{*}$-coloring. If all the colors do not appear in the neighborhood of $x$, then we color $x$ properly. Otherwise, we choose a color for $x$ that does not appear on the $z_{i}$ 's ; there are at least two such colors. If one of these colors is of type $O_{j}$, say $O_{1}$, then we color $x$ with $O_{1}$ and recolor the light small neighbors colored with $O_{1}$ as in the proof of Claim 4. So assume the possible colors for $x$ are of type $D_{i}$. We color $x$ with one of these colors that appears at most $d$ times on the light small neighbors of $x$, say $D_{1}$ (such a color exists, for otherwise $k \geq a+b+2 d$ ). It remains to recolor properly the light small neighbors of $x$ that see $d+1$ times the color $D_{1}$. This yields a $(d, a, b)^{*}$-coloring of $G$, a contradiction.

\subsection{Discharging procedure}

Set

$$
\begin{aligned}
r_{1} & =\frac{d(a+1)}{(a+d+1)(a+1)+a b}, \\
r_{2} & =\frac{d a}{(a+d+1)(a+1)+a b}, \\
\bar{\delta} & =a+b+\frac{d a(a+1)}{(a+d+1)(a+1)+a b} .
\end{aligned}
$$

We apply a discharging procedure to prove that no graphs of maximum average degree less than $\bar{\delta}$ satisfy Claims 1 to 5 (that will show that our minimum couterexample $G$ to Theorem 4 cannot exist). We first assign to each vertex $v$ a charge $\omega(v)$ equal to its degree, $\forall v \in V(G), \omega(v)=d_{G}(v)$. We then apply the discharging rules $\mathbf{R} 1$ and $\mathbf{R 2}$ (below). Let $\omega^{*}(v)$ be the new charge of the vertex $v$ once the discharging procedure is finished. We will prove that, for every vertex $v, \omega^{*}(v) \geq \bar{\delta}$. Since before the discharging procedure, the average charge is less than $\bar{\delta}$, and after it is at least $\bar{\delta}$, we reach a contradition.

Let us now prove that for all $v, \omega^{*}(v) \geq \bar{\delta}$.

The rules are the following:

R1. Every non-small vertex gives $r_{1}$ to each adjacent light small vertex.

R2. Every non-small vertex gives $r_{2}$ to each adjacent small vertex that is not light.

Consider a vertex $v$ of degree $k$. By Claim $1, k \geq a+b$. We distinguish three cases:

- $v$ is small. By Claim 2, $v$ is adjacent to at least $a$ non-small vertices. If $v$ is adjacent to exactly $a$ non-small vertices, then $v$ is light by definition and so it receives $a \cdot r_{1}$ by R1. Hence

$$
\omega^{*}(v)=k+a \cdot r_{1} \geq a+b+a \cdot r_{1}=\bar{\delta} .
$$


If $v$ is adjacent to at least $a+1$ non-small vertices, $v$ receives at least $(a+1) \cdot r_{2}$ by $\mathrm{R} 2$. Hence

$$
\omega^{*}(v) \geq k+(a+1) \cdot r_{2} \geq a+b+(a+1) r_{2}=\bar{\delta} .
$$

- $v$ is medium. By Claim 3, $v$ is adjacent to at least $a-1$ non-small vertices. By Claim 5, $v$ is adjacent to at most $k-a-b+1$ light small vertices. From R1 and R2, together with the fact that $r_{1} \geq r_{2}$, we get the following:

$$
\begin{aligned}
\omega^{*}(v) & \geq k-(k-a-b+1) r_{1}-(a+b-1-(a-1)) r_{2} \\
& \geq a+b+d-(d+1) r_{1}-b r_{2}=\bar{\delta} .
\end{aligned}
$$

- $v$ is big. By Claim 4, $\mathrm{R} 1$ and $\mathrm{R} 2$,

$$
\begin{aligned}
\omega^{*}(v) & \geq k-(k-b) r_{1}-b r_{2} \\
& \geq a+b+2 d-(a+2 d) r_{1}-b r_{2}=a+b+\frac{d(a+1)(a+2)+d a b}{(a+d+1)(a+1)+a b}>\bar{\delta} .
\end{aligned}
$$

Hence, for all $v$ in $V(G), \omega^{*}(v) \geq \bar{\delta}$ as claimed. This completes the proof of Theorem 4 .

\section{Proof of Theorem 5}

To prove Theorem 5, we first give the construction of the graph $G_{d, a, b}$. Then we show that it is not $(d, a, b)^{*}$-colorable, but it is $(d, 0, a+b+1)^{*}$-colorable. Finally, we compute its maximum average degree.

We construct the graph $G_{d, a, b}$ by recursion on $a$. For $a=0$, we set $G_{d, 0, b}=K_{b+1}$, the complete graph on $b+1$ vertices. Suppose now $a \geq 1$. We first define a graph $F_{x}$ (called the fan on $x$ ) as follows : take $d+1$ disjoint copies of $G_{d, a-1, b}$ (denoted $H_{1}, \ldots, H_{d+1}$ ), and add a vertex $x$ adjacent to all the vertices of every copy. To form $G_{d, a, b}$, now take $b+1$ fans $F_{x_{1}}, \ldots, F_{x_{b+1}}$, and form a complete graph on $x_{1}, \ldots, x_{b+1}$. The graph $G_{d, a, b}$ is depicted in Figure 1 .

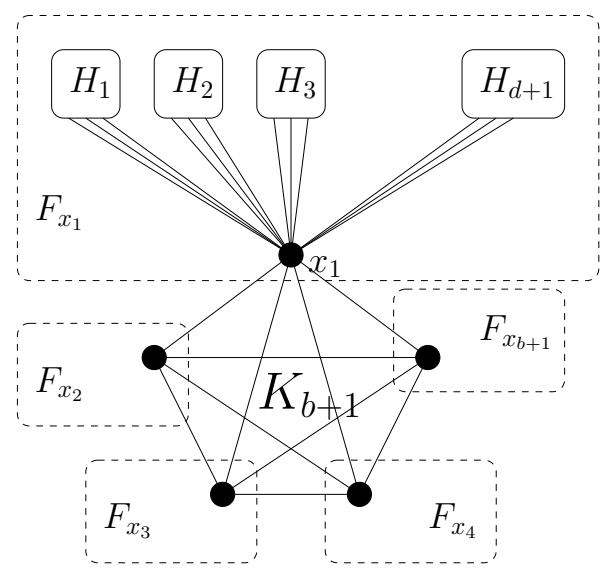

Figure 1: The graph $G_{d, a, b}$.

Claim 6 The graph $G_{d, a, b}$ is not $(d, a, b)^{*}$-colorable. 
PROOF. We prove this claim by induction on the value of $a$. For $a=0, G_{d, a, b}=K_{b+1}$ is clearly not $b$-colorable. Let $a \geq 1$, suppose the graph $G_{d, a, b}$ is $(d, a, b)^{*}$-colorable, and consider such a coloring. By construction, in each fan, the copies $H_{k}$ of the graph $G_{d, a-1, b}$ are not $(d, a-1, b)^{*}$ colorable. So in our coloring, each copy $H_{k}$ must contain at least one vertex colored with each color $D_{i}(1 \leq i \leq a)$. The vertex $x$ thus has $d+1$ neighbors in each color class $D_{i}$, and must be colored with a color of type $O_{j}(1 \leq j \leq b)$. Therefore, the coloring of the subgraph induced by $\left\{x_{1}, \ldots, x_{b+1}\right\}$ must be a proper $b$-coloring, a contradiction.

Claim 7 The graph $G_{d, a, b}$ is $(d, 0, a+b+1)^{*}$-colorable, i.e. properly $(a+b+1)$-colorable.

PROOF. We prove the result by induction on $a$. For $a=0, G_{d, a, b}=K_{b+1}$ is clearly $(b+1)$ colorable. For some $a \geq 1$, assume now that $G_{d, a-1, b}$ is properly $(a+b)$-colorable. Let $x_{1}, \ldots, x_{b+1}$ be defined as in the construction of $G_{d, a, b}$. We color each copy of $G_{d, a-1, b}$ in the fan $F_{x_{k}}$ using the $a+b$ colors in $\{1, \ldots, a+b+1\} \backslash\{k\}$, which is possible by induction. Then, we color each vertex $x_{k}$ with color $k$, and we get a $a+b+1$ proper coloring of $G_{d, a, b}$.

We now prove that the maximum average degree of $G_{d, a, b}$ is given by $\mathfrak{g}(d, a, b)$. Our proof is by induction on $a$, and to simplify notations, we consider in the following that $d$ and $b$ are fixed. The following claims give the number of vertices and edges of $G_{d, a, b}$. With Claim 10, we prove that the maximum average degree is equal to the average degree and give its value.

Claim 8 The number of vertices of $G_{d, a, b}$ is given by

$$
n_{a}=(b+1) \frac{(d+1)^{a+1}(b+1)^{a+1}-1}{(d+1)(b+1)-1} .
$$

PROOF. By construction, we have $n_{0}=b+1$ and $n_{a+1}=(b+1)\left((d+1) n_{a}+1\right)=(b+1)(d+$ 1) $n_{a}+n_{0}$. We recognize here a geometric series of ratio $(b+1)(d+1)$. The result follows from standard arithmetic.

Claim 9 The number of edges of $G_{d, a, b}$ is given by

$$
e_{a}=(b+1) \frac{(d+1)^{a+1}(b+1)^{a+1}\left(\left(a+\frac{b}{2}\right)((d+1)(b+1)-1)-1\right)-\left(\frac{b}{2}-1\right)(d+1)(b+1)+\frac{b}{2}}{((d+1)(b+1)-1)^{2}} .
$$

This is obtained by computing the sequence $e_{a}$ defined recursively by $e_{0}=\frac{b(b+1)}{2}$ and $e_{a+1}=$ $(d+1)(b+1)\left(e_{a}+n_{a}\right)+\frac{b(b+1)}{2}$. The detailed computation is given in the Annex.

Claim 10 The maximum average degree of $G_{d, a, b}$ is attained on the whole graph, and is equal to $\mathfrak{g}(d, a, b)$ defined by

$$
\mathfrak{g}(d, a, b)=2 a+b-\frac{2}{(d+1)(b+1)-1}+\frac{2 a+2}{(d+1)^{a+1}(b+1)^{a+1}-1} .
$$

Proof. We prove this by induction on $a$. First note that for $a=0, G_{d, a, b}=K_{b+1}$ and has a maximum average degree of $b$ attained on the whole graph.

We denote the average degree of a graph $G$ by $\mu(G)=\frac{2|E|}{|V|}$. We show that any subgraph $G^{\prime}$ of $G_{d, a, b}$ satisfies $\mu\left(G^{\prime}\right) \leq \mu(G)$, and thus that $\operatorname{mad}(G)=\mu(G)$. Note that we can consider only induced subgraph, which have maximum average degree for a given subset of vertices.

Let $a \geq 1$ and suppose that the claim is true for all $G_{d, a^{\prime}, b}$ when $a^{\prime}<a$. Consider a connected induced subgraph $G^{\prime}$ of $G_{d, a, b}$. Let $\beta \leq b+1$ be the number of vertices in $G^{\prime}$ from the central clique $\left\{x_{1}, \ldots, x_{b+1}\right\}$. Let $t$ be the number of non-empty copies of $H=G_{d, a-1, b}$ in $G^{\prime}$ (i.e. copies such that $\left.V(H) \cap V\left(G^{\prime}\right) \neq \emptyset\right), n_{1}, \ldots, n_{t}$ their orders and $\mu_{1}, \ldots, \mu_{t}$ their average-degrees. We assume that $\beta$ and $t$ are positive. Remark that, since $G^{\prime}$ is connected, we have $t \leq \beta(d+1)$. 
The average degree of $G^{\prime}$ can be computed by

$$
\begin{aligned}
\mu\left(G^{\prime}\right) & =\frac{2 E\left(G^{\prime}\right)}{V\left(G^{\prime}\right)}=\frac{2\left(\sum_{i=1}^{t}\left(\frac{1}{2} \mu_{i} n_{i}+n_{i}\right)+\frac{1}{2} \beta(\beta-1)\right)}{\sum_{i=1}^{t} n_{i}+\beta} \\
& =\frac{\sum_{i=1}^{t}\left(\mu_{i}+2\right) n_{i}+\beta(\beta-1)}{\sum_{i=1}^{t} n_{i}+\beta}
\end{aligned} .
$$

Applying this to $G$ we get (with $\mu_{H}=\mu(H), n_{H}=|V(H)|$, and $r=(d+1)(b+1)$ )

$$
\mu(G)=\frac{r\left(\mu_{H}+2\right) n_{H}+b(b+1)}{r n_{H}+b+1} .
$$

The proof that $\mu(G) \geq \mu\left(G^{\prime}\right)$ is given in the Annex.

\section{References}

[1] K. Appel and W. Haken. Every planar map is four colorable. Part I. Discharging. Illinois J. Math., 21:429-490, 1977.

[2] K. Appel and W. Haken. Every planar map is four colorable. Part II. Reducibility Illinois J. Math., 21:491-567, 1977.

[3] O.V. Borodin and A.O. Ivanova. Near proper 2-coloring the vertices of sparse graphs. Diskretn. Anal. Issled. Oper., 16(2):16-20, 2009.

[4] O.V. Borodin, A.O. Ivanova, M. Montassier, P. Ochem, and A. Raspaud. Vertex decompositions of sparse graphs into an edgeless subgraph and a subgraph of maximum degree at most $k . J$. Graph Theor., 65(2):83-93, 2010.

[5] O.V. Borodin, A.O. Ivanova, M. Montassier, and A. Raspaud. $(k, 1)$-coloring of sparse graphs. Discrete Math., 312(6):1128-1135, 2012.

[6] O.V. Borodin, A.O. Ivanova, M. Montassier, and A. Raspaud. $(k, j)$-coloring of sparse graphs. Discrete Appl. Math., 159(17):1947-1953, 2011.

[7] O.V. Borodin and A.V. Kostochka. Vertex partitions of sparse graphs into an independent vertex set and subgraph of maximum degree at most one. Sibirsk. Mat. Zh., 52(5):1004-1010, 2011 (in Russian).

[8] L.J. Cowen, R.H. Cowen, and D.R. Woodall. Defective colorings of graphs in surfaces: partitions into subgraphs of bounded valency. J. Graph Theor., 10(2):187-195, 1986.

[9] N. Eaton and T. Hull. Defective list colorings of planar graphs. Bull. Inst. Combin. Appl., 25:79-87, 1999.

[10] L. Esperet, M. Montassier, P. Ochem, and A. Pinlou. A complexity dichotomy for the coloring of sparse graphs. To appear in J. Graph Theor., 2012.

[11] A.N. Glebov and D.Zh. Zambalaeva. Path partitions of planar graphs. Sib. Èlektron. Mat. Izv., http://semr.math.nsc.ru, 4:450-459, 2007 (in Russian).

[12] F. Havet and J.-S. Sereni. Improper choosability of graphs and maximum average degree. $J$. Graph Theor., 52:181-199, 2006.

[13] R. Škrekovski. List improper coloring of planar graphs. Comb. Proba. Comput., 8:293-299, 1999.

[14] R. Škrekovski. List improper coloring of planar graphs with prescribed girth. Discrete Math., 214:221-226, 1999. 


\section{Annex}

Proof of Claim 9. Let $r=(d+1)(b+1)$. We have to compute here the terms of the sequence given by

$$
\left\{\begin{aligned}
e_{0} & =\frac{b(b+1)}{2} \\
e_{a+1} & =(d+1)(b+1)\left(e_{a}+n_{a}\right)+\frac{b(b+1)}{2} \\
& =r\left(e_{a}+n_{a}\right)+e_{0}
\end{aligned}\right.
$$

We use another sequence $F_{a}$ defined by

$$
F_{a}=e_{a}+U_{a} r^{a+1}+C
$$

where $U_{a}$ is some sequence and $C$ some constant value yet to define. The idea here is to choose them so that $F_{a}$ is a geometric progression of ratio $r$. We have $F_{a+1}=e_{a+1}+U_{a+1} r^{a+2}+C$, which we want equal to $r F_{a}$. Using the recursive relation for $e_{a+1}$ and the computed value of $n_{a}$, namely $n_{a}=(b+1) \frac{r^{a+1}-1}{r-1}$, we get:

$$
\begin{aligned}
F_{a+1} & =e_{a+1}+U_{a+1} r^{a+2}+C \\
& =r\left(e_{a}+(b+1) \frac{r^{a+1}-1}{r-1}+U_{a+1} r^{a+1}\right)+C+e_{0} \\
& =r\left(e_{a}+r^{a+1}\left(\frac{b+1}{r-1}+U_{a+1}\right)\right)-\frac{r(b+1)}{r-1}+C+e_{0} \\
& =r\left(e_{a}+r^{a+1}\left(U_{a+1}+\frac{b+1}{r-1}\right)+C\right)-r C-\frac{r(b+1)}{r-1}+C+e_{0}
\end{aligned}
$$

In order to get $F_{a+1}=r F_{a}=r\left(e_{a}+U_{a} r^{a+1}+C\right)$, we set

$$
\begin{aligned}
U_{a+1} & =U_{a}-\frac{b+1}{r-1} \\
C & =\frac{1}{r-1}\left(e_{0}-\frac{r(b+1)}{r-1}\right)=\frac{(r-1) e_{0}-r(b+1)}{(r-1)^{2}}
\end{aligned}
$$

Setting $U_{0}=0$, we obtain $U_{a}=-a \frac{b+1}{r-1}$ and $F_{a}=F_{0} r^{a}$. It follows that:

$$
\begin{aligned}
e_{a} & =\left(e_{0}+C\right) r^{a}-U_{a} r^{a+1}-C \\
& =r^{a}\left(e_{0}+\frac{(r-1) e_{0}-r(b+1)}{(r-1)^{2}}+a r \frac{b+1}{r-1}\right)-\frac{(r-1) e_{0}-r(b+1)}{(r-1)^{2}} \\
& =\frac{r^{a}\left(r(r-1) e_{0}-r(b+1)+a r(r-1)(b+1)\right)-(r-1) e_{0}+r(b+1)}{(r-1)^{2}} \\
& =\frac{r^{a+1}\left((r-1) \frac{b}{2}(b+1)+(b+1)(a(r-1)-1)\right)-(r-1) \frac{b}{2}(b+1)+r(b+1)}{(r-1)^{2}} \\
& =(b+1) \frac{r^{a+1}\left(\left(a+\frac{b}{2}\right)(r-1)-1\right)-\left(\frac{b}{2}-1\right) r+\frac{b}{2}}{(r-1)^{2}}
\end{aligned}
$$

Replacing $r$ by $(d+1)(b+1)$, we get

$$
e_{a}=(b+1) \frac{(d+1)^{a+1}(b+1)^{a+1}\left(\left(a+\frac{b}{2}\right)((d+1)(b+1)-1)-1\right)-\left(\frac{b}{2}-1\right)(d+1)(b+1)+\frac{b}{2}}{((d+1)(b+1)-1)^{2}}
$$

Proof OF Claim 10. We prove that $\mu(G) \geq \mu\left(G^{\prime}\right)$ to conclude the proof of Claim 10. We use again the notation $r=(d+1)(b+1)$. 
We compute

$$
R=\left(\mu(G)-\mu\left(G^{\prime}\right)\right)\left(r n_{H}+b+1\right)\left(\sum_{i=1}^{t} n_{i}+\beta\right)
$$

and show it is non-negative, using the values of $\mu(G)$ and $\mu\left(G^{\prime}\right)$ obtained in the main part of the proof of Claim 10. We obtain

$$
\begin{aligned}
R= & \left(r\left(\mu_{H}+2\right) n_{H}+b(b+1)\right)\left(\sum_{i=1}^{t} n_{i}+\beta\right) \\
& -\left(\sum_{i=1}^{t}\left(\mu_{i}+2\right) n_{i}+\beta(\beta-1)\right)\left(r n_{H}+b+1\right) \\
= & r\left(\mu_{H}+2\right) n_{H} \sum_{i=1}^{t} n_{i}-r n_{H} \sum_{i=1}^{t}\left(\mu_{i}+2\right) n_{i}+\beta b(b+1)-\beta(\beta-1)(b+1) \\
& +r\left(\mu_{H}+2\right) n_{H} \beta-r n_{H} \beta(\beta-1)-(b+1) \sum_{i=1}^{t}\left(\mu_{i}+2\right) n_{i}+b(b+1) \sum_{i=1}^{t} n_{i} \\
= & r n_{H}\left(\sum_{i=1}^{t}\left(\mu_{H}-\mu_{i}\right) n_{i}\right)+\beta(b+1)(b+1-\beta) \\
& +(b+1) \sum_{i=1}^{t}\left(b-\left(\mu_{i}+2\right)\right) n_{i}-\beta \sum_{i=1}^{r}\left((\beta-1)-\left(\mu_{H}+2\right)\right) n_{H}
\end{aligned}
$$

By induction, $\mu_{H} \geq \mu_{i}$. Moreover $\beta \leq b+1$. Thus the first line of the last expression is clearly non-negative. Let $R^{\prime}=(b+1) \sum_{i=1}^{t}\left(b-\left(\mu_{i}+2\right)\right) n_{i}-\beta \sum_{i=1}^{r}\left((\beta-1)-\left(\mu_{H}+2\right)\right) n_{H}$ be the expression on the last line. We have

$$
\begin{aligned}
R^{\prime}= & (b+1) \sum_{i=1}^{t}\left(b-\left(\mu_{i}+2\right)\right) n_{i}-\beta \sum_{i=1}^{t}\left((\beta-1)-\left(\mu_{H}+2\right)\right) n_{i} \\
& -\beta \sum_{i=1}^{t}\left((\beta-1)-\left(\mu_{H}+2\right)\right)\left(n_{H}-n_{i}\right)-\beta \sum_{i=t+1}^{r}\left((\beta-1)-\left(\mu_{H}+2\right)\right) n_{H}
\end{aligned}
$$

Again, since $\mu_{H} \geq \mu_{i}$ and $\beta \leq b+1$, the first line is non-negative. By construction, $H$ contains a clique of order $b+1$ and by induction, its maximum average degree is equal to its average degree. Thus, $\mu_{H} \geq b \geq \beta-1$. Clearly, we have $\mu_{H}+2 \geq \beta-1$ and thus the second line is non-negative. Therefore, $R$ is non-negative, which proves the first part of our claim.

To compute $\operatorname{mad}\left(G_{d, a, b}\right)$, we now simply have to compute $\frac{2 e_{a}}{n_{a}}:$

$$
\begin{aligned}
\frac{2 e_{a}}{n_{a}} & =\frac{2(b+1) \frac{r^{a+1}\left(\left(a+\frac{b}{2}\right)(r-1)-1\right)-\left(\frac{b}{2}-1\right) r+\frac{b}{2}}{(r-1)^{2}}}{(b+1) \frac{r^{a+1}-1}{r-1}} \\
& =\frac{(2 a+b)(r-1) r^{a+1}-2 r^{a+1}-(b-2) r+b}{\left(r^{a+1}-1\right)(r-1)} \\
& =\frac{(2 a+b)(r-1)\left(r^{a+1}-1\right)+(2 a+b)(r-1)-2\left(r^{a+1}-1\right)-2-b(r-1)+2 r}{\left(r^{a+1}-1\right)(r-1)} \\
& =2 a+b-\frac{2}{r-1}+\frac{2 a+2}{r^{a+1}-1}=\mathfrak{g}(d, a, b)
\end{aligned}
$$

